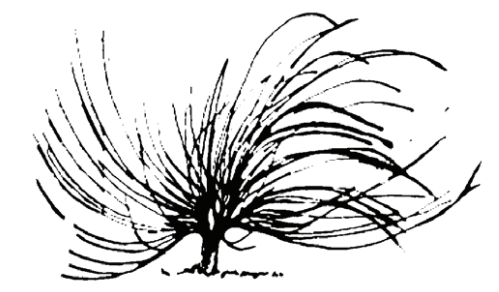

\title{
Apreciaciones del Estudiantado con Respecto al Desempeño de su Docente de Ciencias Naturales
}

\author{
Helen Navarro Castrol \\ Ministerio de Educación Pública \\ San José, Costa Rica \\ hellen.karina.navarro@gmail.com
}

\section{Resumen}

Este artículo tiene como propósito dar a conocer algunas apreciaciones del estudiantado de secundaria con respecto al desempeño de sus docentes de ciencias; los resultados expuestos fueron obtenidos a partir de la aplicación de dos instrumentos: a) una visita de aula, en la cual se observó a tres docentes hasta obtener saturación de datos y b) un cuestionario dirigido a estudiantes de III ciclo, donde los informantes indicaron algunas apreciaciones de sus docentes de ciencias en cuanto a su desempeño en el trabajo de aula. El marco teórico contempla tópicos de la Política Educativa vigente emitida por el Ministerio de Educación Pública. La metodología empleada se ubica en una tradición propia del paradigma naturalista, aunque contempla la recolección, el análisis y la vinculación de datos cualitativos y cuantitativos en un mismo estudio. Entre los principales hallazgos está la relevancia que posee el proceso de comunicación para el logro de un buen aprendizaje, así como la importancia de contar

Recibido: 30 de setiembre de 2013 - Aprobado: 18 de agosto de 2014

1 Actualmente labora como docente de Ciencias en el Liceo Dr. José María Castro Madriz, posee 13 años de experiencia en el campo docente. Máster en Educación con énfasis en Administración Educativa de la Universidad de Costa Rica y Licenciada en la Enseñanza de las Ciencias de la Universidad Nacional. 
con un ambiente de aula donde prevalezca el respeto y la cooperación entre otros.

Palabras clave: política educativa vigente, mediación pedagógica, aprendizaje significativo, apreciaciones, desempeño docente

\begin{abstract}
This paper aims to report some findings about secondary students' appraisal of their Science teacher's performance; the displayed results were obtained from the application of two instruments: a) a visit to the classroom, in which three teachers were observed until data saturation was achieved and b) a questionnaire for students of third cycle, in which the respondents indicated their appraisal of their science teachers' performance in the classroom. The theoretical framework includes topics about the current Educational Policy issued by the Ministry of Education. The methodology is located in the tradition of the naturalistic paradigm, but it includes the collection, analysis and linking of qualitative and quantitative data in the same study. Among the key findings of this research, the relevance of the communication process to achieve good learning stands out along with the importance of having a classroom where respect and cooperation among others prevail.
\end{abstract}

Keywords: current educational policy, pedagogical mediation, meaningful learning, appraisal, teacher performance

\title{
Introducción
}

$\mathrm{E}$ 1 presente artículo se fundamenta en los resultados de la investigación llamada "Perfil del Desempeño de los profesionales de la Enseñanza de las Ciencias en III Ciclo, de un Colegio del Circuito 03 de la Dirección Regional de San José Central", presentada en agosto del año 2012. El artículo se sustenta en las apreciaciones que tiene el estudiante con respecto al desempeño de su profesor de Ciencias Naturales en su trabajo de aula, donde prevalece la buena comunicación clara y con lenguaje sencillo; aunado a lo anterior, es necesario que 
en las lecciones, la medicación pedagógica propicie espacios de índole participativa; así como estimular la creatividad e iniciar procesos de investigación con trabajos pequeños, como los que se implementan en las ferias científicas tanto a nivel institucional como regional.

Por tanto, este artículo es de gran utilidad para futuros estudiantes de la carrera de Enseñanza de las Ciencias Naturales, por ende, a los docentes en servicio para que reflexionen acerca de algunas demandas expuestas por los estudiantes y con ello mejorar nuestra mediación pedagógica.

\section{Antecedentes}

En agosto del año 2012, previa a la exposición de la investigación antes mencionada, "Perfil del Desempeño de los profesionales de la Enseñanza de las Ciencias en III Ciclo, de un Colegio del Circuito 03 de la Dirección Regional de San José Central”, no se halló referencias relacionadas con el artículo que actualmente se lee; sin embargo, considero pertinente citar aspectos vinculados con el tema antes expuesto.

Para iniciar, el Ministerio de Educación Pública (2005), en su papel de institución rectora de la formación académica, persigue como objetivo central la ejecución de planes y programas que emanan del Consejo Superior de Educación, dentro de los que destaca la Política Educativa Vigente hacia el Siglo XXI, sustentada en la Constitución Política de Costa Rica y la Ley Fundamental de Educación, la cual fue aprobada por el Consejo Superior de Educación en sesión No 82-94, el 8 de noviembre de 1994, siendo Ministro de Educación el Dr. Eduardo Doryan.

La Política Educativa se nutre de tres fuentes filosóficas llamadas humanismo, racionalismo y constructivismo, las cuales deben estar presentes tanto en los contenidos (el que), como en los procesos cognoscitivos (el cómo) y en el desarrollo de valores (el para qué) que se relacionan de forma simultánea. Dentro de esta política, se destaca un apartado llamado continuidad e innovación de acciones educativas que fue modificado por el Consejo Superior de Educación en la sesión 77-90, del 16 de octubre, 1990, donde se indica: "El educador debe ser el facilitador, colaborador y orientador del aprendizaje del educando" (p. 8); no obstante, el docente de ciencias además de su conocimiento en pedagogía debe ser conocedor de las diferentes áreas de las ciencias, a saber, biología, química y física, aunado a la actualización de conocimientos de ellas. 
De modo similar, el Tercer Estado de la Educación (2011, p. 81), señala que el docente debe generar ambientes de aprendizaje atractivos y de calidad, que potencien el desarrollo de los estudiantes, a su vez satisfagan sus necesidades; por tanto, el buen desempeño del profesional en las ciencias naturales debe contemplar diversidad en metodologías de acuerdo con la temática vista y, al mismo tiempo, motivar al estudiantado y tomar en cuenta sus ideas para enlazarlas al nuevo contenido.

Lo expresado en los párrafos anteriores, se vincula con el desempeño del profesor de ciencias en cuanto aspira a tener una visión social de la educación, concibiéndose así mismo como una transformador social donde considere las diferencias individuales y emplee diferentes estilos y ritmos de aprendizaje.

\section{Justificación}

Con el paso de los años, los docentes costarricenses han experimentado cambios en su quehacer de aula, ya que el mismo va más allá de planear y ejecutar las lecciones correspondientes; por otro lado, a los docentes de ciencias se les ha encomendado la organización y planificación de la Feria Científica, la cual busca que los alumnos exploren su potencial científico. Por tanto, la labor del docente en la enseñanza de las ciencias no es fácil:

$[\mathrm{M}]$ muchas veces debe romper con estigmas y tabúes y desmitificar la ciencia, partiendo de crear, en una fase inicial, una visión global de todo acontecimiento científico, que a pesar de estar focalizado al momento de su descubrimiento, sus repercusiones serán políticas, económicas y a la vez socioculturales siempre con consideraciones éticas de por medio. (Saborío, 2001, p. 44)

Saborío (2004, p. 45) indica que la visión innovadora de la ciencia por parte del profesorado requiere del compromiso de someterse a un cambio actitudinal, de renovarse en sus preconcepciones de cómo enseñar ciencias y no resistirse a lo nuevo.

En el IV Informe de investigación del Proyecto Perfiles, Dinámicas y Desafíos de la Educación Costarricense del CIDE - UNA, se indica que los docentes se preocupan por dar lo mejor de sí mismos en su labor, por lo que es muy importante que tengan muy claro el rol que 
desempeñan como docentes y la relevancia de esta profesión, la cual debe estar acompañada de un buen manejo del vocabulario, presentación personal impecable, buenas relaciones humanas, buena ortografía y buena expresión oral.

Los docentes deben aspirar a tener una visión social de la educación, concibiéndose a sí mismos como transformadores sociales, lo cual incide en su realidad como profesionales:

El (la) educador(a) nace pero también se hace; no es solo lograr títulos que les permitan adquirir un mejor status, debe existir un compromiso por parte de todos(as) los(as) participantes en los procesos educativos para contribuir con el desarrollo humano de las personas que se están formando así como en la transformación social. (Martínez, Alfaro, Badilla, Calderón, Martín y Vargas, 2009, p. 148).

Por tanto, se requiere para el III ciclo un(a) docente que:

- $\quad$ Tenga conciencia de la naturaleza heterogénea que caracteriza al ser humano y acepte a cada persona sin reparar en su condición física, social, económica, cultural, ni en sus creencias religiosas, filosóficas, afiliaciones deportivas, políticas, recreativas, entre otros.

- $\quad$ Tome en consideración las diferencias individuales así como la interculturalidad en la mediación pedagógica en relación con la capacidad intelectual, motora, artística y creativa, entre otras, y adoptar una posición flexible para poner en práctica una variedad de metodologías que le permitan emplear distintos elementos visuales, auditivos, prácticos y concretos, los cuales, según el caso, complementa con experiencias en las que se utilicen otros sentidos, como el tacto, propiciando el aprendizaje de acuerdo con los diferentes estilos y ritmos de aprendizaje e inteligencias múltiples de los(as) estudiantes. (Martínez et al., 2009, p. 132).

Hoy, es necesario escuchar y atender las apreciaciones de los estudiantes hacia el desempeño de los docentes en pro de una buena relación interpersonal, así como la transmisión de conocimientos de manera significativa y con ello forjar un proceso de enseñanza y de aprendizaje de calidad. 
Para efectos de este artículo, debe entenderse como calidad en educación el generar ambientes de aprendizaje propios para la adquisición de destrezas, habilidades, actitudes y valores (Tercer Estado de Educación, 2011, p. 35).

\section{Problema}

¿Cuáles son algunas de las apreciaciones del estudiantado con respecto al desempeño de su docente de ciencias naturales?

\section{Objetivo General}

Analizar el desempeño del docente de ciencias a la luz de lo que propone la Política Educativa Vigente.

\section{Objetivos Específicos}

Describir las características del docente de ciencias naturales desde la apreciación del estudiante.

Caracterizar el desempeño profesional del docente de ciencias naturales.

\section{Marco Teórico}

El presente apartado retoma aspectos que se vinculan con el desempeño de los docentes de ciencias que laboran para el Ministerio de Educación Pública que orienta el desarrollo del proceso educativo costarricense, entre ellos:

\section{A. Política educativa vigente}

La Política Educativa se caracteriza por una concepción de "transdisciplinariedad", donde las disciplinas de naturaleza científica tradicional y las ciencias sociales y humanas se interconectan y se afectan mutuamente. Por tanto, el concepto de persona se nutre de tres visiones filosóficas contempladas en el marco legal vigente:

a.1) Humanista, como la base para la búsqueda de la plena realización del ser humano, de la persona dotada de dignidad y valor, capaz de procurar su perfección mediante la realización de los 
valores estipulados en la legislación educativa, tanto los de orden individual como los de carácter social.

a.2) Racionalista, como el reconocimiento de que el ser humano está dotado de una capacidad racional que puede captar objetivamente la realidad en todas sus formas, construir y perfeccionar de continuo los saberes y hacer posible el progreso humano el entendimiento entre las personas.

a.3) Constructivista, como el esfuerzo en el actuar considerando que la educación debe partir desde la situación cognoscitiva del alumno, de su individualidad, de sus intereses e idiosincrasia, por lo que debe reconocer la cultura específica del alumno con sus respectivas estructuras de conocimiento ya formadas y emprender una acción formativa del alumno y del conocimiento que los transforme mutuamente.

De acuerdo con la Política Educativa vigente, el docente debe abordar, en forma sólida, los conocimientos, los procesos de construcción y reconstrucción de conocimientos. Desde este contexto, los programas de estudio deben ser una guía relevante para el docente desde el punto de vista de la planificación de su trabajo de mediación en coherencia con los recursos didácticos, el proceso de evaluación y sus propias necesidades e intereses profesionales.

\section{B. Mediación pedagógica}

Para Valverde (2004, p. 22), la mediación es una forma de interacción pedagógica que conduce el principal dispositivo de aprendizaje y desarrollo emocional de las personas: "Así los docentes de Ciencias en su papel de mediadores pedagógicos se constituyen en facilitadores del aprendizaje y el desarrollo de habilidades científicas" (Valverde, 2004, p. 23).

Para efectos de este artículo, debe entenderse como mediación pedagógica el logro de una buena comunicación e interacción entre docente - alumno y el contexto en el que se encuentran; para ello es importante citar aspectos de carácter formativo como el aseo y orden del aula y el planeamiento elaborado por el docente debe contemplar los diversos estilos de aprendizaje (visual, auditivo, kinestésico) aunado a 
los recursos tecnológicos como D.V.D, T.V., entre otros. En un ambiente de aula es vital la participación del estudiante para detectar logros y debilidades dentro del proceso enseñanza y aprendizaje.

\section{Aprendizaje significativo}

Díaz y Hernández (2010, p. 31) señalan que el aprendizaje significativo en el aula implica un procesamiento activo de la información por aprender; es decir, cuando se relacionan ideas nuevas con las previas y se reorganizan conocimientos a partir de la estructura cognitiva propia de cada individuo; para lograrlo, es necesario que se den condiciones como la disposición o actitud por aprender además de la estructura y organización del contenido.

En el campo de la enseñanza de las ciencias naturales, se requiere que los docentes empleen una metodología basada en diversos puentes cognitivos como mapas conceptuales, analogías, entre otros, que le permitan al estudiantado ahondar más sobre una temática y generar en este la criticidad.

\section{Metodología}

\section{Tipo de investigación}

Con base en los objetivos propuestos, la investigación se ubica en el paradigma naturalista, la cual señala que es "el estudio de los significados de las acciones humanas y de la vida social y se centra en que la realidad es dinámica, múltiple, holística" (Dobles, 2001, p. 98).

Así mismo, esta investigación utilizó una metodología que combina el uso de técnicas cualitativas y cuantitativas para la recolección de información, metodología que Hernández, Fernández y Baptista (2007), han de llamar mixta y que permite recolectar, analizar y vincular datos cualitativos y cuantitativos en un mismo estudio o en una serie de investigaciones para responder al planteamiento del problema.

\section{Fases de la investigación}

La investigación se llevó a cabo en tres fases, a saber: 


\section{Fase. Visitas de aula}

Es la fase inicial, se realizaron visitas de aula hasta obtener saturación de datos y se visualizaron algunas características del desempeño profesional que presentan los docentes de ciencias en su práctica. El instrumento utilizado se dividió en dos partes: la primera constó de información general y la segunda contempló aspectos de tipo formativos, planeamiento, mediación pedagógica y psicopedagógica, por último, un espacio para que el observador brindara observaciones generales.

\section{Fase. Aplicación de cuestionarios a estudiantes}

Para esta segunda fase, se seleccionó una muestra escogida por el docente de aula, tomando en cuenta que sus estudiantes presentaran buen comportamiento y además atendieran indicaciones.

Se entiende como "muestra", un grupo de personas, eventos, sucesos, comunidades, etc., donde se recolectan los datos (Hernández, Fernández y Baptista 2007, p. 562). La muestra seleccionada la conformaron dos grupos de sétimo año y una igual cantidad de grupos de octavo y noveno año, para un total de 87 alumnos y un promedio de 29 discentes por sección de un colegio académico diurno.

\section{Fase. Análisis de Resultados}

En esta tercera y última fase, se realizó un análisis tanto de las visitas de aula como de los cuestionarios aplicados a los estudiantes. Las visitas de aula se analizaron de forma cualitativa mediante un informe basado en la construcción de un instrumento. Para ello, se consideraron aspectos del planeamiento, mediación tanto pedagógica como psicopedagógica y se seccionó por docente, de acuerdo con el nivel impartido en las lecciones. Es importante aclarar que no se realizó el mismo número de observaciones para cada docente, ya que dependían de la repetición de datos observados y tabulados. Es importante también señalar que las observaciones de aula no fueron en igual cantidad para los docentes que participaron, ya que ellas dependían de si existía repetición o saturación de datos. Tanto para la docente de sétimo como para el de octavo, se realizaron cuatro visitas de aula no participantes, mientras que al docente de noveno año se le realizaron tres.

Los cuestionarios se analizaron de dos formas: a) la primera y segunda parte del instrumento de forma cuantitativa acompañado de la construcción de algunos gráficos; b) y la tercera parte del instrumento 
de forma cualitativa representada por algunas matrices. Los cuestionarios fueron analizados por nivel, sintetizando criterios emitidos por los alumnos (as) que respondieron el instrumento.

\section{a) Cuestionario a los estudiantes de Tercer Ciclo}

El cuestionario se define como un conjunto de preguntas, sean estas cerradas o abiertas; las primeras consisten en delimitar la respuesta, puede ser dicotómica (dos respuestas) o que existan varias opciones de respuesta (Hernández, Fernández y Baptista 2007, p. 310) y las segundas, proporcionan una información más amplia y sirven en situaciones donde se desea profundizar una opinión (Hernández, Fernández y Baptista, 2007, p. 316). Para este estudio, se les entregó a los estudiantes de la muestra un cuestionario con preguntas cerradas y abiertas sobre las características que consideraban deben tener los docentes de ciencias en su desempeño profesional.

El cuestionario mencionado se estructuró en dos secciones: en la primera, el estudiante debía brindar información sobre su género y nivel en que se encontraba matriculad y la segunda sección constaba de tres partes: la primera consistía en marcar con una equis Si o No, las primeras siete preguntas; en la segunda parte, el estudiante escogía según su criterio en una escala que va de uno a tres, a cada uno de los enunciados que se le presentan, a partir de la pregunta ocho hasta la pregunta veintidós, y una última parte que constaba de cuatro preguntas de carácter abierto, en las cuales se invitaba al estudiante a dar su criterio en relación con el desempeño de su profesor de Ciencias.

\section{Descripción de los sujetos de investigación}

\section{a) Fuente institucional}

El presente estudio se centró en el Departamento de Ciencias de un colegio Académico Diurno, conformado por 1219 estudiantes, divididos en 17 sétimos con 546 alumnos matriculados, 9 octavos conformados por 294 estudiantes, 7 novenos con 245, 5 décimos con 175 y 6 undécimos con 175 alumnos. 


\section{b) Fuente humana}

\section{b. 1 Docentes Departamento de Ciencias}

Para este estudio, se realizaron visitas de aula no participantes con el fin de no intervenir en el ambiente de aula, participaron tres docentes.

\section{b.2. Estudiantes del Tercer Ciclo del área de Ciencias}

Para este caso, se tomará como muestra dos grupos de sétimo, octavo y noveno año.

\section{Descripción del análisis de información}

Una vez obtenido los resultados de cada instrumento, se procedió a realizar un análisis general, en el cual se extrajeron y se agruparon por categorías las distintas características que describían el desempeño del docente de ciencias. Por tanto, la utilización de múltiples métodos permite desarrollar una investigación sistemática, a su vez, genera un estudio completo en sí mismo (Hernández, Fernández y Baptista 2007, p. 790).

\section{Resultados, análisis y discusión}

Uno de los aspectos que se investigó con respecto al desempeño del docente es su capacidad de comunicación, entendida como un proceso que logra estimular al alumno, facilitar la información pertinente, integrar y dar respuesta a sus solicitudes, crear espacios abiertos de conocimiento, percatarse de situaciones como desinterés, distracción, desatención; entre otros (Calderón, 2002).

Específicamente, se consultó a los estudiantes con respecto a la atención que los profesores brindan a las dudas que puedan generarse ante una situación nueva de conocimiento. De acuerdo con la figura 1, es el docente de noveno quien presta menos atención a las dudas de los estudiantes. 
Figura 1. Docentes de ciencias que atienden dudas sobre la materia

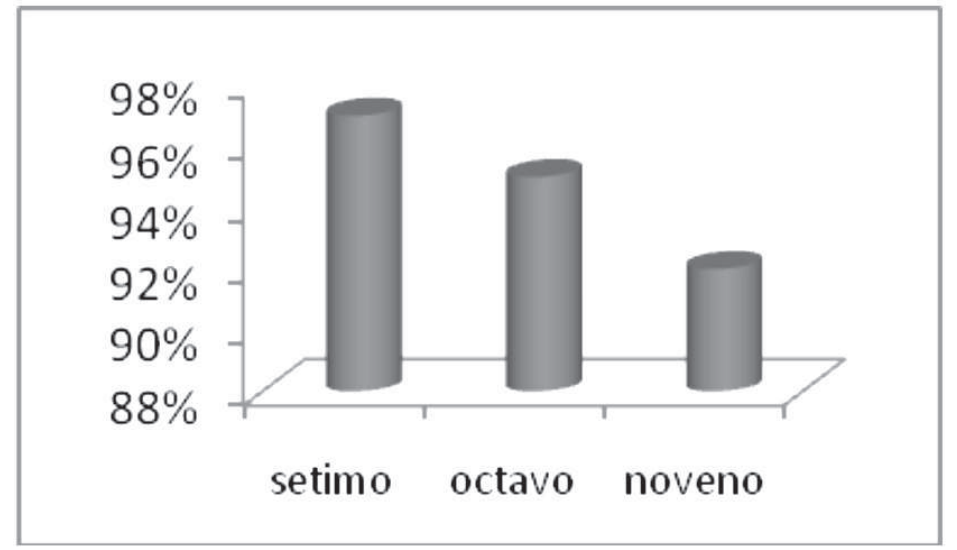

Fuente: Instrumento aplicado a los estudiantes de III Ciclo de un Colegio Público Diurno del Circuito O3 de la Dirección Regional de San José Central. 2011.

En la figura 2, se observa como la docente de ciencias de sétimo año, es la que más genera un ambiente creativo en el salón de aula, entendiéndose como una estrategia para aprender la información, según Díaz - Hernández (2010).

Figura 2. Docentes de ciencias que generan un ambiente creativo en el salón de aula

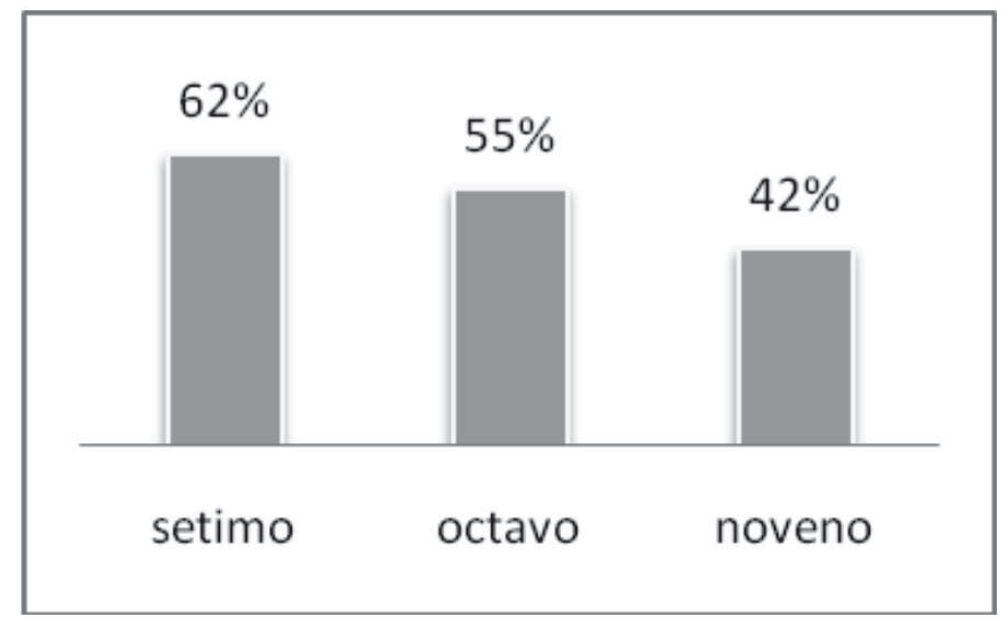

Fuente: Instrumento aplicado a los estudiantes de III Ciclo de un Colegio Público Diurno del Circuito O3 de la Dirección Regional de San José Central. 2011

Según Díaz - Hernández (2010), en el plano pedagógico, incentivar significa estimular la voluntad de aprender y en el contexto 
escolar, permite en gran medida que los alumnos inviertan su atención y esfuerzo en determinadas tareas, que se involucran en las actividades académicas, además implica diversas componentes, cognitivos, afectivos, sociales y académicos con las actuaciones de los alumnos como de los profesores (p. 52). Lo anterior se visualiza en la figura 3, la cual muestra que la docente de sétimo año promueve más la participación de los estudiantes en el salón de aula.

Figura 3. Docentes de ciencias que incentivan la participación de los estudiantes en el salón de aula

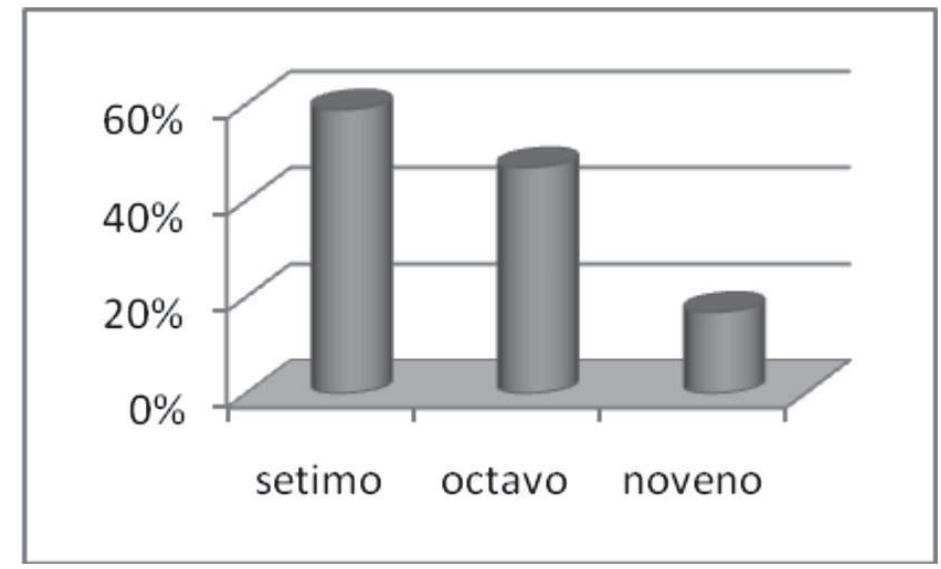

Fuente: Instrumento aplicado a los estudiantes de III Ciclo de un Colegio Público Diurno del Circuito O3 de la Dirección Regional de San José Central. 2011

Los resultados de las observaciones de aula se incorporaron en la tabla 1. En ella se describen aspectos de mediación pedagógica y psicopedagógica. 
Tabla 1. Observación de aula de los docentes de ciencias de III Ciclo de un Colegio Público Diurno del Circuito 03 de la Dirección Regional de Educación de San José Central

\begin{tabular}{|c|c|c|c|}
\hline \multicolumn{4}{|c|}{ Docente } \\
\hline Criterios & 7 & 8 & 9 \\
\hline Mediación pedagógica & $\begin{array}{l}\text { Al estudiante: } \\
\text { Lo motiva, incentiva } \\
\text { la participación en } \\
\text { clase, le atiende } \\
\text { inquietudes } \\
\text { y estimula su } \\
\text { creatividad. }\end{array}$ & $\begin{array}{l}\text { Al estudiante: } \\
\text { Le incentiva la } \\
\text { participación en } \\
\text { clase, le atiende } \\
\text { inquietudes, se le } \\
\text { estimula el proceso de } \\
\text { la investigación con } \\
\text { trabajos pequeños. } \\
\text { El docente: realizó } \\
\text { presentaciones en } \\
\text { PowerPoint y utiliza } \\
\text { recursos tecnológicos } \\
\text { como: video beam, } \\
\text { computadora. }\end{array}$ & $\begin{array}{l}\text { Al estudiante: } \\
\text { Poca intervención; } \\
\text { por tanto no hay } \\
\text { cuestionamientos de la } \\
\text { temática vista en clase; } \\
\text { sino más bien el docente } \\
\text { hace uso excesivo del } \\
\text { libro de texto. }\end{array}$ \\
\hline $\begin{array}{l}\text { Mediación } \\
\text { psicopedagógica }\end{array}$ & $\begin{array}{l}\text { Logra mantener la } \\
\text { atención y el interés } \\
\text { de los alumnos en } \\
\text { la clase. En relación } \\
\text { con los estilos } \\
\text { de aprendizaje } \\
\text { predomina lo visual } \\
\text { y algunas veces } \\
\text { contempla actividades } \\
\text { que considere las } \\
\text { inteligencias múltiples. }\end{array}$ & $\begin{array}{l}\text { Logra mantener la } \\
\text { atención y el interés } \\
\text { de los alumnos en } \\
\text { la clase. Dentro del } \\
\text { estilo de aprendizaje } \\
\text { el que predomina es el } \\
\text { visual y pocas veces } \\
\text { contempla actividades } \\
\text { que considere las } \\
\text { inteligencias múltiples. }\end{array}$ & $\begin{array}{l}\text { Algunas veces logra } \\
\text { mantener la atención } \\
\text { y el interés de los } \\
\text { alumnos en la clase. } \\
\text { En cuanto al estilo } \\
\text { de aprendizaje que } \\
\text { predomina es el } \\
\text { visual y no considera } \\
\text { las inteligencias } \\
\text { múltiples. }\end{array}$ \\
\hline
\end{tabular}

Fuente: Instrumento aplicado a los docentes que imparten ciencias de III Ciclo en colegio público del circuito 03 de la Dirección Regional de Educación de San José Central. 2011.

En los aspectos formativos, es pertinente que en el aula donde se imparten las lecciones se mantenga limpia, ordenada y se sienta un ambiente de interés y de motivación tanto para los alumnos como para el docente y con ello apropiarse del concepto de ecología de aula, el cual atiende aspectos tanto físicos (dimensiones, aireación, aspecto estético, mantenimiento y supervisión del estado del aula) como de "apropiación y pertenencia (apropiación del espacio del aula como exposición de trabajos, noticias opiniones de los alumnos)" (Alfaro et al., 2008, p. 65).

La mediación pedagógica es el tratamiento de contenidos y de las formas de expresión de los diferentes temas a fin de hacer posible que el acto educativo debe ser participativo, creativo, expresivo y 
racionalizado a partir de la construcción de conocimientos, de la investigación y del intercambio de experiencias; tal y como se indica en la matriz, los tres docentes emplean recursos distintos para que el alumno entienda, a excepción del docente de noveno que utiliza en exceso el libro de texto. Por tanto, no permite que los educandos puedan ser críticos o generen conocimiento.

La mediación psicopedagógica debe concebirse como una forma de apropiar el aprendizaje, mediante la motivación (intrínseca o extrínseca) que supla las necesidades del estudiantado y con ello mantener interés de conocer su entorno, contemplando para ello las inteligencias múltiples de los estudiantes y su estilo de aprendizaje, entendiéndose como la forma de aprender ya sea visual, auditivo o kinestésico.

\section{Conclusiones}

Los alumnos de sétimo caracterizan a su docente de ciencias como una persona que se preocupa por enseñarles y destacan como factor importante en su desempeño profesional el incentivarlos a participar de las lecciones.

Con respecto al desempeño de los docentes participantes, ellos utilizan diversas metodologías, según el tema visto en el momento de la observación; sin embargo, es rescatable indicar que la docente que imparte sétimo año es muy dedicada en su trabajo y una cualidad que resalta en ella es la paciencia hacia sus estudiantes.

Dentro de las características apreciadas por los estudiantes, se destacan la atención pronta y oportuna de los docentes; tanto el de sétimo como el de octavo responden a las inquietudes de los estudiantes.

En general, estos docentes deben utilizar distintos estilos de aprendizaje para atender las necesidades de los estudiantes, ya que los tres sólo emplean el estilo visual. Es importante señalar que en la enseñanza de las ciencias naturales debe estimularse todos los estilos de aprendizaje para que la ciencia sea vista de forma dinámica y entretenida. 


\section{Referencias bibliográficas}

Alfaro, M., Gamboa, A., Jiménez, S., Martín, J., Ramírez, A., Vargas, M y Zúñiga, A. (2008). El perfil integrado emergente de desempeño docente para sétimo año. Centro de Investigación y Docencia en Educación. Universidad Nacional.

Alfaro, M., Gamboa, A., Jiménez, S., Martín, J., Ramírez, A., Vargas, M y Zúñiga, A. (2011). Perfil docente: Fundamentos teóricos y metodológicos. Centro de Investigación y Docencia en Educación. Universidad Nacional.

Díaz, F. y Hernández., G. (2010). Estrategias docentes para un aprendizaje significativo._México, Distrito Federal: Mc Graw - Hill Interamericana.

Dobles, M., Zúñiga M. y García, J. (2001). Investigación en educación: Procesos, interacciones, construcciones. San José, Costa Rica: Universidad Estatal a Distancia.

Hernández, R (2007). Metodología de la investigación (4ta Ed.) México. D.F: Mc Graw Hill.

Ministerio de Educación Pública. (2005). Programas de estudio Ciencias III Ciclo. San José, Costa Rica: Autor.

Martínez, J. Alfaro, M. Badilla, A. Calderón, E. Martín y J. Vargas, M. C. (2009). Perfiles dinámicas y desafíos de la educación costarricense. Centro de Investigación y Docencia en Educación. Universidad Nacional.

Programa Estado de la Nación (2011). Tercer informe estado de la educación. San José, Costa Rica: Autor.

Valverde, M. (2004). Mediación en el aula: Desarrollo de un manual de recursos, estrategias y técnicas didácticas para el logro de un aprendizaje significativo en la asignatura de Ciencias de noveno año. [Tesis de Licenciatura no publicada]. Universidad Estatal a Distancia, San José, Costa Rica. 